\title{
On Luxury and Equilibrium
}

\author{
ANDREA MANTOVI \\ Università degli Studi di Parma*
}

\begin{abstract}
Building on a class of transcendental preferences for luxury, explicit solutions for price taking behaviour and exchange equilibrium are discussed, which share the analytical tractability of Cobb-Douglas models. Such economies display fundamental positive properties, among which uniqueness and price tâtonnement stability of equilibrium. The monotone comparative statics of the luxury effect is discussed. Pareto sets admit a simple characterization, which generalizes the one set forth by Afriat (1987) so that a richer phenomenology is embraced. Potential lines of progress are envisaged.
\end{abstract}

JEL classification: D50; D51; D58.

Keywords: Edgeworth Box; General Equilibrium; Luxury; Necessity; Monotone Comparative Statics; Pareto Set.

\section{Introduction}

The theoretical analysis of general equilibrium, on both positive and normative grounds, has long been recognized as the structural problem of economic theory, and in fact as a cogent representation of the decentralized mechanisms supporting the working of Adam Smith's "invisible hand" for competitive market economies. That being the case, "the equations of equilibrium constitute the center of our discipline” (Mas-Colell et al., 1995, p. 620), and the relevance follows of having explicit solutions to such equations. In such respects, the solutions for pure exchange economies with Cobb-Douglas (CD) agents discussed by Afriat (1987) represent a landmark. It is the aim of the present contribution to set forth a class of explicit solutions for Walrasian demand and exchange equilibrium with two agents, built on transcendental utility functions over a pair of commodities, which contains the solutions for CD agents, and shares the nice properties of such models, in first instance, uniqueness and (price tâtonnement) stability of equilibrium, together with the pleasant analytical tractability which justifies to a large extent the pervasiveness of the CD analytic form, "perhaps the most ubiquitous function in all of economics” (Chambers, 1988). We shall employ the Edgeworth

\footnotetext{
*Via J. F. Kennedy 6, 43125 Parma, Italy, andrea.mantovi@unipr.it

(C) 2014 Andrea Mantovi; . Licensed under the Creative Commons Attribution - Noncommercial 3.0 Licence (http://creativecommons.org/licenses/by-nc/3.0/. Available at http: //rofea.org.
} 
box as playground for our analysis of exchange equilibrium, on account of the generality of the insights which can be thereby conveyed. ${ }^{1}$

The vision underlying the present contribution posits that the luxury-necessity dichotomy represents quite general a trait of preferences with respect to the benchmark case of homothetic preferences, in which income (wealth) effects display scale symmetry. Expansion paths of homothetic consumers are rays, so that the relative composition of optimal consumption bundles is unaffected by income effects. With respect to such a benchmark framework, the luxury-necessity dichotomy posits an elementary grain of generality, namely, expansion paths bending (globally) towards luxury, and maintaining the normality of both (possibly, aggregates of) goods. In such respects, our model of transcendental preferences provides a highly tractable representation of the luxury-necessity dichotomy with asymptotic satiation of necessity, i.e. vanishing necessity share in the large expenditure limit.

The benchmark nature of homothetic ${ }^{2}$ models has long been recognized, with CD models representing, in many senses, an ideal ${ }^{3}$ setting. One is led to envision a theoretical 'hierarchy' in the space of preferences, such that homothetic models stand out, among which CD models represent a preferred setting. Definitely, in between the homothetic CD benchmark and the general problem, we shall discuss the well behaviour of a class of preferences for luxury, which combine the analytical tractability of CD models with a significant degree of generality. Noticeably, the parameterization of our preferences enables one to identify a monotone "luxury effect", which one can address in terms of monotone comparative statics, along the lines tailored by Milgrom and Shannon (1994). In addition, the explicit solution for Pareto sets enables one to envision a phenomenology which generalizes the one set forth by Afriat (1987).

Beyond the relevance of our demand functions for empirical analysis ("specific functional forms for aggregate demand relationships are the starting point for a large body of empirical work"; Sonnenshein, 1972), our model strikes a definite perspective on the positive theory of general equilibrium (PTGE). Recall, the Sonnenschein-Mantel-Debreu theorem (see Mas-

1 As Mas-Colell et al. (1995) put it, “There are virtually no phenomena or properties of general equilibrium exchange economies that cannot be depicted in it” (ivi, p. 521). The introduction of the Edgeworth box can be attributed to Pareto (Binmore, 2007).

2 According to Chambers and Mitchell (2001), "Homotheticity may be the most common functional restriction employed in economics.” See Mantovi (2013a, 2013b) for a differential geometric approach to the benchmark scale invariance of homothetic problems. Scale invariance lies at the basis, for instance, of the invariance problem of index numbers, for which Samuelson and Swamy (1974) set forth a landmark analysis.

${ }^{3}$ Consider for instance the fundamental role of CD functions in the class of self-dual (utility or production) functions (Sato, 1976; 1981). In addition, consider the rationale for introducing super Cobb-Douglas utility and production functions (Mas-Colell, 1991), which enrol for instance CES functions with elasticity of substitution coefficient geq 1. For application of the CD functional form as matching function, see Silva and Toledo (2013) and references therein. 


\section{ANDREA MANTOVI On Luxury and Equilibrium}

Colell et al., 1995, and references therein) establishes that, as for excess demand, "anything goes”: given an acceptable aggregate excess demand function (i.e. which satisfies continuity, homogeneity of degree 0 and Walras law), there exist individual excess demand functions from which the aggregate function can be derived. ${ }^{4}$ As a consequence, the theoretical relevance is by now undisputed of establishing significant lines along which to introduce structure on excess demand. For instance, Quah (2003) exploits the normality of goods in the comparative statics of general equilibrium. Definitely, we shall employ the luxury-necessity dichotomy in order to put structure on Marshallian and excess demand functions via the parameterization of transcendental utility functions.

Freixas and Mas-Colell (1987) establish the relevance of the "uniform curvature" condition for the validity of the weak axiom of revealed preference (Samuelson, 1938; "WARP") for aggregate demand. "With normal goods an interpretation is that commodities admit the same classification as necessities or luxuries at any level of admissible income" (ivi, p. 516). True, the Authors allow such a classification to be price dependent, so that a commodity may be a luxury at a given price vector, and a necessity at another. Definitely, our explicit solutions for price taking behaviour and equilibrium provide a transparent realization of the setting discussed by Freixas and Mas-Colell (1987), with no restriction concerning the distribution of income. ${ }^{5}$ Evidently, our approach is 'orthogonal' to the geometric approaches to PTGE (see for instance the classical contribution by Debreu, 1976, and the recent survey by Balasko and Tvede, 2010), in that the precise (flexible) functional form of our preferences is crucial for the following arguments.

The plan of the rest of the paper is as follows. In section 2 we define our exchange economies. In section 3 we represent price taking behaviour and characterize equilibrium, and then sketch the positive relevance of the results. In section 4 we establish the monotone comparative statics of the luxury effect. In section 5 we characterize the Pareto sets of our economies. A final section envisions potential lines of progress. The Appendix collects a number of plots meant to sharpen intuition about the results established in the main text.

\section{A Class of Exchange Economies}

Consider the class of preferences represented by the ordinal utility functions

$$
U_{a, \varepsilon}(x, y)=x^{a}\left(y e^{\varepsilon y}\right)^{1-a}
$$

\footnotetext{
${ }^{4}$ In a sense, such a result establishes the 'completeness' of the language of PTGE.

5 See for instance Hildenbrand (1983) and Chiappori (1985) for the relevance of the distribution of income with respect to the Law of Demand.
} 
over commodities $x$ and $y$. The parameter $a \in(0,1)$ generalizes the corresponding CD parameter, in that the functional form (1) reduces to the CD form for $\varepsilon \rightarrow 0$. The parameter $\varepsilon \in[0, \infty)$ injects increasing luxury into commodity $y$ : the functional form (1) factorizes a term generating a "luxury effect", namely, $F(y)=\exp (\varepsilon(1-a) y)$, such that $d F / d y$ is proportional to $F$, thereby enhancing the analytical tractability of the model. The relevance has been long established of such objective functions in production analysis as transcendental functions (Halter et al., 1957; Mundlak, 1964), a subclass of generalized power functions (de Janvry, 1972). The preferences represented by (1) ${ }^{6}$ are smooth, strongly increasing and strictly convex over the strictly positive quadrant, and we are thereby guaranteed that Marshallian demand is a function 7 and that expansion paths do foliate smoothly the consumption set $(0, \infty) \times(0, \infty)$.

Indifference curves for the preferences (1) can be represented as

$$
x(y ; u)=u^{\frac{1}{a}}\left(y e^{\varepsilon y}\right)^{\frac{a-1}{a}}
$$

Generalizing CD models, such curves approach asymptotically the axes, as represented in Figure 1 by sample curves, for a $=0.75$ and $\varepsilon=0.4$, which embody the "luxury effect" generated by $\varepsilon$ : MRS vary smoothly along rays so that, the larger the bundle, the less luxury is needed to substitute8 a definite amount of necessity.

Let us sketch the basic elements of the consumption model (Mantovi, 2013(b)) which can be straightforwardly translated to the exchange problem via the logic of price taking. The FOC

$$
\text { MRS } \equiv \frac{\frac{\partial U}{\partial U}}{\frac{\partial U}{\partial x}}=\frac{1-a}{a} \frac{x}{y}(1+\varepsilon y)=\frac{p_{y}}{p_{x}} \quad \Rightarrow \quad x p_{x}=\frac{a}{1-a} \frac{y p_{y}}{1+\varepsilon y}
$$

generalize the corresponding CD condition (see for instance Varian, 1992) and provides a Cartesian equation for expansion paths.

Freixas and Mas-Colell (1987) define the uniform curvature (UC) condition, according to which, in essence, expansion paths are required to be either concave or convex. By (3), UC is satisfied by our model. Needless to say, formula (3) is independent of the utility

\footnotetext{
6 To the author's knowledge, such preferences have not been employed in the analysis of general equilibrium.

7 Recall that, in general, Marshallian demand is a correspondence (Afriat, 1987; Mas-Colell et al., 1995)

8 “At the margin, all goods consumed are perfect substitutes” (Silberberg, 2008, p. 20).
} 


\section{ANDREA MANTOVI On Luxury and Equilibrium}

representation; for instance, it can be obtained from the representation $a \ln x+(1-a) \ln y+(1-$ a) $\varepsilon y$ of utility, equivalent to (1).

\section{Figure 1}

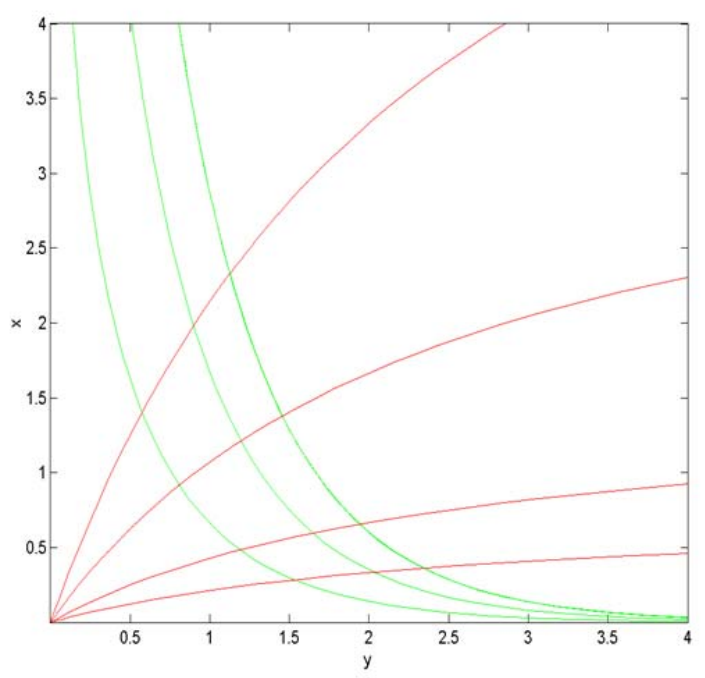

Sample indifference (green) curves with utility levels 2, 4, 6 for the agent (1) with $a=3 / 4$ and $\varepsilon=0.4$, together with expansion (red) paths for $p_{x} / p_{y}=1,2,5,10$.

For positive $\varepsilon$ expansion paths (hyperbolas) bend towards the luxury axis and display asymptotes $\quad x=\beta\left(\frac{p_{y}}{p_{x}}, \varepsilon\right) \equiv \frac{a}{1-a} \frac{p_{y}}{\varepsilon p_{x}}$ corresponding to satiation of necessity; as expected, for given prices, the larger $\varepsilon$, the lower the level $\beta$ of satiation of necessity.

Assume all income is spent. Plug (3) into the budget constraint and obtain

$$
I=x p_{x}+y p_{y}=y p_{y}\left(1+\frac{a}{1-a} \frac{1}{1+\varepsilon y}\right)
$$

from which we derive Marshallian demand for $y$ as solution to the quadratic equation

$$
\varepsilon y^{2}+\left(\frac{1}{1-a}-\varepsilon \frac{I}{p_{y}}\right) y-\frac{I}{p_{y}}=0
$$


Notice, $I$ and $p_{y}$ enter such an expression via their ratio, so as to comply with homogeneity of degree 0 in prices and income. Equation (5) admits a positive solution (the relevant one) and a negative solution; one can check that the positive solution $y^{*}\left(p_{y}, I\right)$ displays income elasticity exceeding 1 for positive $\varepsilon$, and approaches the corresponding CD solution in the limit $\varepsilon \rightarrow 0$. Noticeably, Marshallian demand for $y$ does not depend on $p_{x}$, thereby generalizing the corresponding CD property of vanishing cross-price response. Then, Marshallian demand for $x$ can be written

$$
x^{*}\left(I, p_{x}, p_{y}\right)=\frac{I}{p_{x}}-\frac{p_{y}}{p_{x}} y^{*}\left(I, p_{y}\right)
$$

with the proper CD limit for $\varepsilon \rightarrow 0$, and with non-vanishing cross-price response for $\varepsilon>0$. We can then write the closed form solution for indirect utility $V_{a, \varepsilon}\left(p_{x}, p_{y}, I\right)=U_{a, \varepsilon}\left(x^{*}\left(p_{x}, p_{y}, I\right), y^{*}\left(I, p_{y}\right)\right)$. The simple analytical setting defined by eq. (5) guarantees the existence of explicit solutions for quantities characterizing optimal consumption according to (1). A correspondingly simple analytical setting for exchange can be setup, in which a FOC corresponding to (3) characterizes rational behaviour, and a quadratic equation corresponding to (5) determines Walrasian demand for commodity $y$.

\subsection{Exchange}

The Edgeworth box represents feasible allocations of elementary exchange economies, in which a pair of agents are in a position to exchange a pair of commodities over which they have private ownership, and whose stock we normalize to unity (c.f. Afriat, 1987), in the absence of other meaningful scales. Let the agents A and B belonging to the class (1) be identified by the values $a, \varepsilon$ and $a^{\prime}, \varepsilon^{\prime}$ of the parameters respectively. Let $(x, y)$ denote the quantities of the two commodities owned by agent $\mathrm{A}$, and therefore $(1-x, 1-y)$ those owned by agent B. Agents have initial endowments, which, in general, do not represent a WE. Write $(\bar{x}, \bar{y})$ for the initial endowment of agent A. Let the necessary commodity be the numeraire, so as to let the relative price $p$ define the budget line $x=\bar{x}-p(y-\bar{y})$, which represents bundles with the same value. Let us confine our analysis to the interior $(0,1) \times(0,1)$ of the Edgeworth box, in which both agents are endowed with positive amounts of both commodities, thereby aligning with the assumptions of the first existence theorem set forth by Arrow and Debreu (1954), as well as guaranteeing that our utility functions are strongly increasing on their domain. Such a restriction is consistent, in that the indifference curves (2), generalizing CD models, never hit the boundary of the consumption set $(0, \infty) \times(0, \infty)$. We shall not consider free goods. 


\section{ANDREA MANTOVI On Luxury and Equilibrium}

The extant literature relies heavily on homothetic models in order to address WE. True, despite the theoretical relevance of such models, homothetic consumers cannot be taken to represent general traits of preferences, which in fact the luxury-necessity dichotomy embodied by our model (1) of preferences can accommodate, to some extent. Recall, in fact, the luxury-necessity dichotomy represents a fundamental partition of consumption goods in empirical analyses (Deaton and Muellbauer, 1980).

Consider for instance Engel's Law (see Chakrabarty and Hildenbrand, 2011, for a recent review), according to which the budget share for foodstuff is decreasing with income in the aggregate. Foodstuff is the typical example of necessity, for which empirical analysis has long established the saturation of Engel curves (see for instance Aitchison and Brown, 1954). True, the model (1) of preferences provides a tractable stylized fact accounting for the saturation of consumption of a necessary (possibly, aggregate) commodity, in connection with the asymptotic linearity of Engel curves for an 'associated' luxury (possibly, aggregate) commodity, and then may represent an analytical setting well suited for the analysis of nonbalanced growth. ${ }^{9}$

By the nice properties of the preferences (1), namely, smoothness, strict convexity and strong monotonicity, we are guaranteed the existence of a WE for our economies as a consequence of Proposition 17.C.1 in Mas-Colell et al. (1995). The same properties imply that a price vector is a WE price vector if and only if it is market clearing, i.e. if aggregate excess demand vanishes for each commodity (Mas-Colell et al., 1995, p. 581).

Our analysis is confined to the utility hypothesis, "i.e., the assumption that demand is generated by maximizing a quasi-concave utility function” (Kihlstrom et al., 1976), and we are thereby guaranteed that individual excess demand functions do satisfy WARP. Definitely, WARP represents a strong condition on aggregate excess demand. In fact, by monotonicity of aggregate excess demand one can establish WARP, and then equilibrium price is unique (Mas-Colell et al., 1995). True, being (1) a 'well-behaved' generalization of CD preferences, our economies do satisfy WARP in the aggregate and then admit unique equilibria (section 3).

As for the stability of equilibrium, being $\mathbf{P}$ the price vector, recall the definition $\frac{d \mathbf{P}}{d t}=c \mathbf{z}(\mathbf{P})(c>0)$ of price tâtonnement ${ }^{10}$ stability as a first order ODE defining a pull towards equilibrium prices: a positive (negative) $k$-th component of the excess demand $\mathbf{z}$ leads to increase (decrease) of the corresponding component of $\mathbf{P}$; no pull occurs for vanishing $\mathbf{z}$. One thereby pins down a pregnant stability concept, which can be connected with WARP (for

\footnotetext{
9 To the author's knowledge, the functional form (1) has not been employed to model the demand side of nonbalanced growth, for which the Stone-Geary aggregate is typically employed (Acemoglu, 2008).

${ }^{10}$ See Jaffé (1967) for a thorough discussion of the interpretations of the tâtonnement process (Walras, 1874).
} 
Review of Economic Analysis 6 (2014) 87-118

instance, Arrow et al., 1959; Uzawa, 1960). Definitely, our economies turn out to be stable in such sense.

\section{Walrasian Demand}

\subsection{Parametric Representation}

By the logic of price taking, we are in a position to disentangle the optimization problems of our agents, and reduce to the independent offer problems of two consumers characterized by definite preferences and initial endowments. For each price ratio, each agent has its own best response (offer function) which maximizes his utility on the budget line. Such Walrasian demand function for agent A can be obtained from the solution to the optimization problem

$$
\begin{gathered}
y^{*}(p ; \bar{x}, \bar{y}) \equiv \underset{y \in(0,1)}{\arg \max }\left\{U_{a, \varepsilon}(x, y) \mid x=\bar{x}-p(y-\bar{y})\right\} \\
=\arg \max (\bar{x}-p(y-\bar{y}))^{a} y^{1-a} e^{(1-a) \varepsilon y}
\end{gathered}
$$

with $(\bar{x}, \bar{y}),(x, y) \in(0,1) \times(0,1)$. The solution to problem (7) is uniquely determined by the FOC

$$
\frac{-a p}{\bar{x}-p(y-\bar{y})}+\frac{1-a}{y}+(1-a) \varepsilon=0
$$

which reduces to the quadratic equation

$$
\varepsilon y^{2}+\left(\frac{1}{1-a}-\varepsilon \frac{\bar{x}+p \bar{y}}{p}\right) y-\frac{\bar{x}+p \bar{y}}{p}=0
$$

for Walrasian demand for the luxury commodity. Such a simple equation provides a transparent condition for price taking behaviour, which parallels condition (5) for Marshallian demand. In fact, the term $(\bar{x}+p \bar{y})$ represents the initial wealth of agent $\mathrm{A}$, which corresponds to the income/wealth parameter $I$ in (5). Then, being $p$ the (relative) price of the luxury commodity, eq. (9) can be obtained directly from eq. (5) via the substitution $\frac{I}{p_{y}} \rightarrow \frac{\bar{x}+p \bar{y}}{p}$. In the $\mathrm{CD}$ limit $\varepsilon \rightarrow 0$, eq. (9) reduces to

$$
\frac{p}{1-a} y-(\bar{x}+p \bar{y})=0
$$




\section{ANDREA MANTOVI On Luxury and Equilibrium}

and therefore admits the well-known solution $y=(1-a) \frac{\bar{x}+p \bar{y}}{p}$ (for instance, Mas-Colell et al., 1995, p. 519), which parallels the corresponding expression $y=(1-a) \frac{I}{p_{y}}$ for Marshallian demand (for instance, Varian, 1992, p. 111). Then, for positive $\varepsilon$, the positive solution to (9) reads

$$
y_{\mathbf{A}}^{*}(p ; \bar{x}, \bar{y})=\frac{1}{2}\left(\frac{-1}{\varepsilon(1-a)}+\frac{\bar{x}+p \bar{y}}{p}+\sqrt{\left(\frac{-1}{\varepsilon(1-a)}+\frac{\bar{x}+p \bar{y}}{p}\right)^{2}+4 \frac{\bar{x}+p \bar{y}}{\varepsilon p}}\right)
$$

The corresponding solution $x_{\mathrm{A}}^{*}(p ; \bar{x}, \bar{y})=\bar{x}+p \bar{y}-p y_{\mathrm{A}}^{*}(p ; \bar{x}, \bar{y})$ for the other commodity is then uniquely determined. We thereby establish the parametric representation of the offer curve for agent A originating at the endowment $(\bar{x}, \bar{y})$, for $p$ larger or smaller than the MRS at $(\bar{x}, \bar{y})$.

For the sake of definiteness, call Adam the agent characterized by $a=0.75, \varepsilon=0.4$. Let his initial endowment correspond to exactly half the resources of the economy, i.e. $(\bar{x}=1 / 2, \bar{y}=1 / 2)$. Such a symmetric endowment stands out with respect to general allocations, in that it represents equality of resources for the two agents participating in the exchange economy. Such an allocation is a WE for infinite pairs of agents (1), as will be established in section 5. Still, in general, a pair of agents (1) may find it profitable to exchange fractions of their endowments, as manifested by the following example.

The symmetric endowment corresponds to Adam's utility level being approximately 0.5256 and MRS $=2 / 5$. The function (11) boils down to

$$
y_{\text {Adam }}^{*}\left(p ; \frac{1}{2}, \frac{1}{2}\right)=\frac{1}{2}\left(-10+\frac{1+p}{2 p}+\sqrt{\left(10-\frac{1+p}{2 p}\right)^{2}+5 \frac{1+p}{p}}\right)
$$

We can employ such an expression in order to plot Adam's excess demand $z_{y \text { Adam }}(p ; \bar{x}, \bar{y})=y_{\text {Adam }}^{*}(p ; 0.5,0.5)-0.5$ for the luxury commodity for the endowment $(\bar{x}=1 / 2, \bar{y}=1 / 2)$. Figure 2 (above) represents the monotone character of $z_{y}$ Adam; it is not difficult to convince oneself that the same monotonicity attains for any endowment. Corresponding results hold for the necessary commodity (Proposition 1 below): given (12), 
Review of Economic Analysis 6 (2014) 87-118

$x_{\mathrm{Adam}}^{*}\left(p ; \frac{1}{2}, \frac{1}{2}\right)$ can be identified via the budget line. We thereby determine the parametric representation of the offer curve originating at the initial endowment. For instance, for $p=$ 0.5, formula (12) established Adam's excess demand for the luxury commodity as $y_{\text {Adam }}^{*}\left(\frac{1}{2} ; \frac{1}{2}, \frac{1}{2}\right)-\frac{1}{2} \cong-0.0796$, and then excess demand for the necessary commodity as $x_{\text {Adam }}^{*}\left(\frac{1}{2} ; \frac{1}{2}, \frac{1}{2}\right)-\frac{1}{2} \cong 0.04$, so that Adam has incentives to offer a fraction of his endowment of the luxury commodity in exchange of some of the necessary one, as represented in the upper part of Figure 2.

The same argument can be employed in order to establish the offer curve for agent $\mathrm{B}$, characterized by parameters $a^{\prime}, \varepsilon^{\prime}$ and initial endowment $(1-\bar{x}, 1-\bar{y})$. From equation (9) we obtain

$$
\varepsilon^{\prime}(1-y)^{2}+\left(\frac{1}{1-a^{\prime}}-\varepsilon^{\prime} \frac{1-\bar{x}+p(1-\bar{y})}{p}\right)(1-y)-\frac{1-\bar{x}+p(1-\bar{y})}{p}=0
$$

by means of the substitution $y \rightarrow 1-y$, whose solution parallels the functional form (11). Then, for the agent Bob characterized by $a^{\prime}=0.5, \varepsilon^{\prime}=0.5$, endowed with $(1-\bar{x}=\bar{x}=1 / 2,1-\bar{y}=\bar{y}=1 / 2)$, and therefore with utility approximately 0.566 and MRS = 1.25 , we have

$$
(1-y)_{\text {Bob }}^{*}\left(p ; \frac{1}{2}, \frac{1}{2}\right)=\frac{1}{2}\left(-4+\frac{1+p}{2 p}+\sqrt{\left(-4+\frac{1+p}{2 p}\right)^{2}+4 \frac{1+p}{p}}\right)
$$

from which we derive the excess demand represented in the lower part of Figure 2.

Recall, an agent is said to be a net demander (or supplier) of a commodity at $p$ if his Walrasian demand of that commodity at $p$ exceeds (or is lower than) his initial endowment, i.e. his excess demand for the commodity is positive (or negative). Then, excess demand for a commodity is positive at $p$ if both agents are net demanders, or if net demand of one agent exceeds net supply of the other agent. The relative price $p$ at which net demand equals net supply for a commodity is a clearing relative price. As can be read off Figure 2, it turns out that for both Adam and Bob the initial symmetric allocation $(\bar{x}=1 / 2, \bar{y}=1 / 2)$ can be improved upon by exchange: for $p$ belonging to the interval $(0.4,1.25)$ Adam is a net supplier of the luxury commodity, whereas Bob is a net demander, and a unique relative price $p^{*} \in(0.4,1.25)$ exists which clears both markets. 


\section{ANDREA MANTOVI On Luxury and Equilibrium}

Figure2
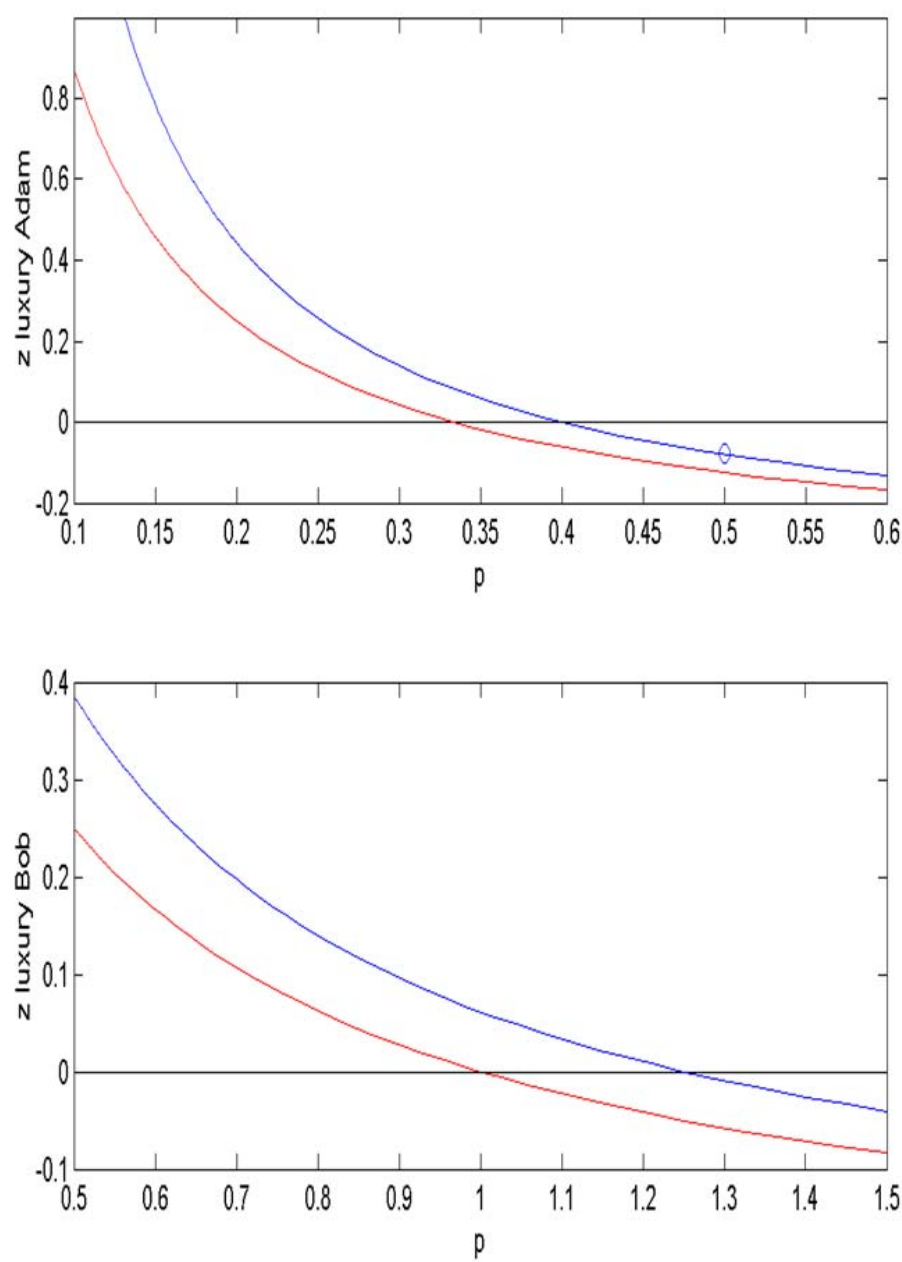

Above. Adam's excess demand (12) for the luxury commodity (blue curve). The function vanishes for $p=0.4$ (MRS at the initial endowment); the dot represents Adam's offer -0.0796 at $p=1 / 2$. The corresponding CD $a=0.75$ excess demand (red curve) $y=(1+p) /(8 p)-0.5$ vanishes at $p=1 / 3$. Below. Bob's excess demand (14) for the luxury commodity (blue curve), vanishing for $\mathrm{p}=$ 1.25. The corresponding CD $\mathrm{a}^{\prime}=0.5$ excess demand (red curve) $y=(1+p) /(4 p)$ -0.5 vanishes at $p=1$. 
Figure 3 represents the aggregate excess demand

$$
\begin{gathered}
z_{\text {luxury }}\left(p ; \frac{1}{2}, \frac{1}{2}\right)=y_{\text {Adam }}^{*}\left(p ; \frac{1}{2}, \frac{1}{2}\right)+\left(1-y_{\text {Bob }}^{*}\right)\left(p ; \frac{1}{2}, \frac{1}{2}\right)-1 \\
=\frac{1}{2}\left(-10+\frac{1+p}{2 p}+\sqrt{\left(10-\frac{1+p}{2 p}\right)^{2}+5 \frac{1+p}{p}}\right)+\frac{1}{2}\left(-4+\frac{1+p}{2 p}+\sqrt{\left(-4+\frac{1+p}{2 p}\right)^{2}+4 \frac{1+p}{p}}\right)-1
\end{gathered}
$$

Figure3

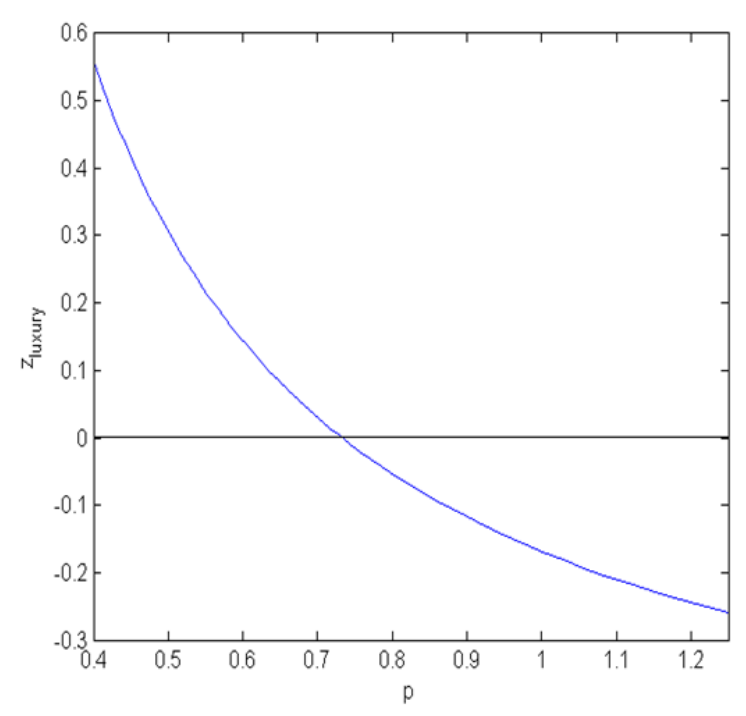

Aggregate Adam-Bob excess demand for the luxury commodity for $p \in(0.4,1.25)$ for the symmetric initial allocation. The function vanishes approximately at 0.73 .

for the luxury commodity for the symmetric initial allocation, which vanishes at $p^{*}$ roughly 0.73. The smooth monotonicity and convexity of the function (15) are displayed in Figure $3^{11}$. The nice behaviour thereby represented extends for all positive $p$, and is not special to the agents and initial allocation considered.

For general agents belonging to the class (1) and for general initial allocation, the aggregate excess demand for the luxury commodity

${ }^{11}$ One can compare Figure 3.2 in Kehoe (1998) representing a case of nonuniqueness of equilibrium. 
ANDREA MANTOVI On Luxury and Equilibrium

$$
\begin{gathered}
z_{\text {luxury }}(p ; \bar{x}, \bar{y})=y_{\mathrm{A}}^{*}(p ; \bar{x}, \bar{y})+\left(1-y_{\mathrm{B}}^{*}\right)(p ; \bar{x}, \bar{y})-1 \\
=\frac{1}{2}\left(\frac{-1}{\varepsilon(1-a)}+\frac{\bar{x}+p \bar{y}}{p}+\sqrt{\left.\left(\frac{-1}{\varepsilon(1-a)}+\frac{\bar{x}+p \bar{y}}{p}\right)^{2}+4 \frac{\bar{x}+p \bar{y}}{\varepsilon p}\right)}\right. \\
+\frac{1}{2}\left(\frac{-1}{\varepsilon^{\prime}\left(1-a^{\prime}\right)}+\frac{(1-\bar{x})+p(1-\bar{y})}{p}+\sqrt{\left(\frac{-1}{\varepsilon^{\prime}\left(1-a^{\prime}\right)}+\frac{(1-\bar{x})+p(1-\bar{y})}{p}\right)^{2}+4 \frac{(1-\bar{x})+p(1-\bar{y})}{\varepsilon^{\prime} p}}\right)-1
\end{gathered}
$$

is smooth, decreasing and convex, and vanishes at the unique equilibrium relative price

$$
p^{*} \equiv z_{\text {luxury }}^{-1}(0)
$$

which clears both markets: needless to say, condition (17) can be formulated in terms of aggregate excess demand for either commodity.

Notice, for increasing $\varepsilon$, the excess demand for the luxury good increases, and we are in a position to define a "luxury effect". In fact for the CD agents $a=0.75$ and $a^{\prime}=0.5$ with symmetric initial allocation $(0.5,0.5)$, equilibrium is attained once their Walrasian demand for the luxury commodity clear, i.e. $\frac{1+p}{8 p}=1-\frac{1+p}{4 p}$, so that equilibrium relative price results in $p^{*}=3 / 5$, and then $y^{*}=1 / 3$ and $x^{*}=3 / 5$. The corresponding equilibrium quantities for Adam and Bob are affected by the interplay of the luxury effects driven by $\varepsilon=0.4$ and $\varepsilon^{\prime}=$ 0.5 , so that the equilibrium relative price is higher than $3 / 5$ (approximately 0.73 ), Adam ends up with $y^{*}>1 / 3$ and $x^{*}>3 / 5$. The luxury effect is clearly depicted in Figure 2, in which positive values of $\varepsilon$ and $\varepsilon^{\prime}$ determine upward shifts of excess demand functions. True, a thorough account of the monotonicity of the luxury effect can be given along the lines tailored by Milgrom and Shannon (1994), which we address in section 4.

To sum up, we have gathered the elements necessary to uniquely identify a WE for any pair of agents (1) and for any internal initial allocation, as well as provide a full-fledged characterization of the well behaviour represented in formulas (11) and (16). 
Review of Economic Analysis 6 (2014) 87-118

PROPOSITION 1. For any pair of agents belonging to the class (1), our exchange economies are such that both individual and aggregate excess demand functions $(0, \infty)$ $\rightarrow \mathrm{R}$ for the luxury commodity are $C^{\infty}$, strictly decreasing in the relative price $p=\frac{p_{y}}{p_{x}}$ and strictly convex for any endowment. Then, both individual and aggregate excess demand for the necessary commodity are $C^{\infty}$, strictly increasing and strictly concave in p for any endowment.

Proof. Compute first and second derivatives of (11) and (16) with respect to $p$ and check the above statement.

We thereby establish the regularity of our economies, as well as the monotonicity of excess demand functions, which represents "the law of demand for any kind of (normalized) price change” (Mas-Colell, 1991, p. 277). Recall the definition of a regular exchange economy (Mas-Colell, et al., 1995) with $n$ commodities. Choose a commodity as numeraire, and consider the aggregate vector excess demand $\zeta$ of the remaining commodities as function of the $n-1$ relative prices $\pi$. The economy is regular if the $(n-1) \times(n-1)$ jacobian matrix $D \equiv \frac{\partial \zeta}{\partial \pi}$ is non-singular, i.e. if the differentials of the $n-1$ components of $\zeta$ are linearly independent over all relative prices. Equivalently, the definition can be given in terms of normalized prices belonging to the unit simplex (Kehoe, 1998). Definitely, for our economies, $n-1=1$, and $D$ reduces to the derivative of (16): thus, by our choice of the necessary commodity as numeraire, the regularity condition boils down to aggregate excess demand for the luxury commodity having positive or negative slope, i.e. not displaying a stationary point. Notice, existence of a stationary point is not incompatible with monotonicity of excess demand, but such critical economy may represent the 'transition' towards nonuniqueness of equilibrium (see Kehoe, 1998, Fig. 3.5 for a representative pattern) and more generally the fact that variations in relative prices may determine variations of excess demand which may not be arbitrarily small (i.e. inverse excess demand non uniformly continuous). On account of the monotonicity embodied by (16), our model maintains the well behaviour shared by CD agents, in particular, aggregate WARP and price tâtonnement stability are satisfied. 


\section{ANDREA MANTOVI On Luxury and Equilibrium}

\subsection{Cartesian Representation}

We have gone a long way in the characterization of our economies, and in fact provided a complete solution to the equilibrium problem, as well as established the nice price taking behaviour represented in Figure 2, 3 and Proposition 1. True, the parametric representation of offer curves is not the only viable approach to the analytics of price taking; the Cartesian representation provides a useful analytical setting as well, for instance with respect to graphical analysis (see Figure 4) which is an invaluable generator of insights. Definitely, capitalizing on the tractability of the preferences (1), we are in a position to derive response functions from the FOC expressing the tangency of indifference curves and budget lines, thereby obtaining a Cartesian representation of offer curves which does not contemplate prices: given price taking behaviour, the condition

$$
\frac{1-a}{a} \frac{x}{y}(1+\varepsilon y)=-\frac{x-\bar{x}}{y-\bar{y}} \quad \Rightarrow \quad x(y-\bar{y})(1+\varepsilon y)=-\frac{a}{1-a} y(x-\bar{x})
$$

establishes the Walrasian demand of agent A. The Cartesian representation

$$
x_{\mathrm{A}}^{*}(y ; \bar{x}, \bar{y})=\frac{\frac{a}{1-a} \bar{x} y}{(y-\bar{y})(1+\varepsilon y)+\frac{a}{1-a} y}
$$

for A's offer curve follows, with the correct limit $\bar{x}$ for $y \rightarrow \bar{y}$. For instance, for the initial endowment $(\bar{x}=1 / 2, \bar{y}=1 / 2)$, Adam's ( $\mathrm{a}=0.75, \varepsilon=0.4)$ offer curve admits the Cartesian representation

$$
x_{\text {Adam }}^{*}\left(y ; \frac{1}{2}, \frac{1}{2}\right)=\frac{3.75 y}{y^{2}+9.5 y-1.25}
$$

plotted in Figure 4. One can check that such a representation is consistent with the previously discussed parametric representation. Again, the simple substitution y $\rightarrow 1-\mathrm{y}$ enables us to write down the Cartesian representation of the offer curve for agent $\mathrm{B}$, namely,

$$
(1-x)_{\mathrm{B}}^{*}(y ; \bar{x}, \bar{y})=\frac{\frac{a^{\prime}}{1-a^{\prime}}(1-\bar{x})(1-y)}{(\bar{y}-y)\left(1+\varepsilon^{\prime}(1-y)\right)+\frac{a^{\prime}}{1-a^{\prime}}(1-y)}
$$

Then, for $\operatorname{Bob}\left(a^{\prime}=0.5, \varepsilon^{\prime}=0.5\right)$ with initial endowment $(1-\bar{x}=1 / 2,1-\bar{y}=1 / 2)$, we have 
Review of Economic Analysis 6 (2014) 87-118

$$
x_{\mathrm{Bob}}^{*}\left(y ; \frac{1}{2}, \frac{1}{2}\right)=1-\frac{\frac{1}{2}(1-y)}{\left(\frac{1}{2}-y\right)(1+0.5(1-y))+(1-y)}=\frac{y^{2}-4.5 y+2.5}{y^{2}-5.5 y+3.5}
$$

The unique WE for the economy defined by Adam and Bob with symmetric initial allocation is represented in Figure 4 at the intersection of offer curves, with $\mathrm{y}^{*}$ approximately 0.32, $\mathrm{x}^{*}$ approximately 0.64 , and equilibrium relative price $\mathrm{p}^{*}$ approximately 0.73 (compare Figure 3 ). We thereby obtain a transparent representation of the well behaviour of our exchange economies. The qualitative pattern represented in Figure 4 holds for all agents (1) and for all initial conditions (endowments) in the open Edgeworth box. Recall, multiplicity of equilibrium is connected with twisty offer curves and turning points in excess demand curves (in the words of Cowell, 2005), phenomena for which our economies make no room (Proposition 1). By means of Figure 4 we can build a solid intuition concerning the mechanism guaranteeing the positive properties of our equilibria, which we deepen via monotone comparative statics in the following section.

Figure 4

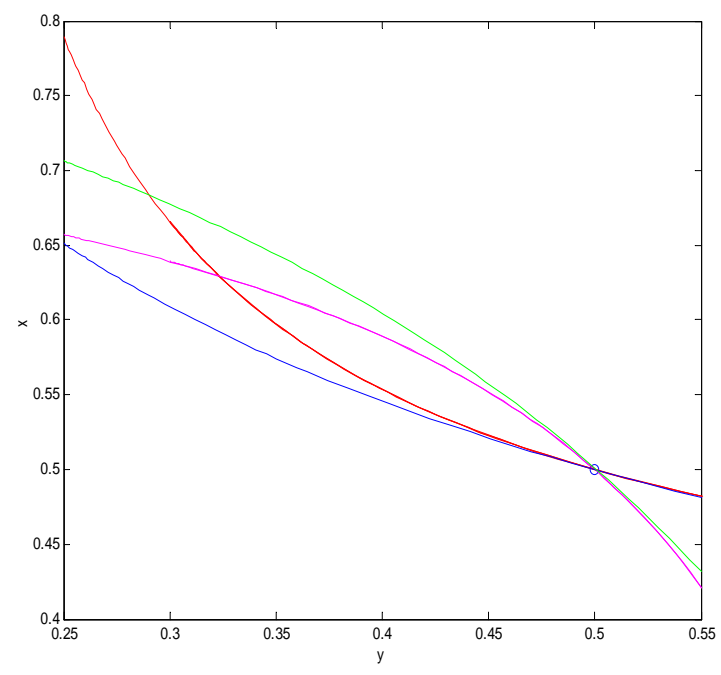

Offer curves for Adam (red) and Bob (magenta) for initial symmetric allocation $(0.5,0.5)$ against indifference curves (blue: Adam, green: Bob) in the subset $(0.4,0.8) \times(0.25,0.55)$ of the Edgeworth box. The intersection of offer curves, at $(x, y) \approx(0.64,0.32)$, identifies the unique WE allocation. Analogously regular patterns hold for all agents (1) and all initial allocations. 


\section{ANDREA MANTOVI On Luxury and Equilibrium}

\section{Luxury Effect}

Finally, armed with a thorough account of the positive properties of our economies, we are in a position to address comparative statics. By the explicit solutions for Walrasian demand established in the previous section, one can employ the parameterization (1) of preferences in order to gauge the effects driving the rational responses of agents, and then the resulting (unique) exchange equilibria. As a matter of fact, any parameter in a model may be taken to define an economic 'effect'; true, the parameter $\varepsilon$ in our utility functions (1) represents a significant monotone trait of rational behaviour, with respect to which we are in a position to address monotone comparative statics along the lines tailored by Milgrom and Shannon (1994, “MS” henceforth).

Figure 5

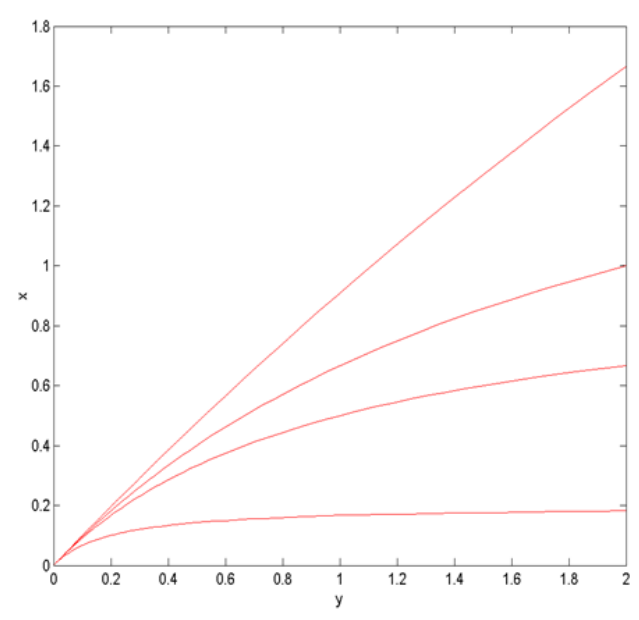

Expansion path $p_{x}=p_{y}$ for the agents (1) with $(a, \varepsilon)=(0.5,0.1$; upper expansion path with satiation level $\beta=10),(0.5,0.5)$, $(0.5,1),(0.5,5$; lower expansion path with satiation level $\beta=0.2$ ). Compare Fig. 1 in which a number of expansion paths are represented for the same agent.

As anticipated in section 3, a luxury effect appears to drive Walrasian demand for the luxury commodity of agents (1) so as to produce monotone upward shifts, for any a, p and initial endowment, as represented in Figure 2. Such a property parallels the property of increasing bending of expansion paths towards the luxury axis established by formula (3); Figure 5 plots the expansion path $\mathrm{px}=$ py for sample values of $\varepsilon$ at fixed a: as intuition suggests, the larger $\varepsilon$, the larger the luxury share for all values of income/wealth. 
Correspondingly, one may ask whether an analogous monotonicity holds for the effect driven by a. For agents belonging to the class (1), call individual distributive effects those generated by a on individual price taking behaviour, and call interplay distributive effect the one generated by both parameters a, a' on the equilibrium of the economy. We employ the term "distributive" on account of the analogy with production analysis. For instance, Fuss et al. (1978, ch. 4) enrol the distributive share effect among the key effects in production12. Interplay distributive effects have been long established as well: it is well known that identical CD preferences (vanishing interplay distributive effect) force a WE to lie on the diagonal of the Edgeworth box (for instance, Afriat, 1987). Definitely, in order to assess the interplay distributive effect, a determination of the Pareto set is necessary (next section).

In order to argue about the monotonicity of such effects, let us follow MS in the setup of comparative statics. For any positive $p$ and any initial endowment $(\bar{x}, \bar{y}) \in(0 \times 1) \times(0 \times 1)$, consider the budget lines $x=\bar{x}-p(y-\bar{y})$, which can be ordered by increasing y so as to represent a lattice.

PROPOSITION 2. For any $p \equiv \frac{p_{y}}{p_{x}}>0$, any $a \in(0,1)$ and any initial allocation, our agents display excess demand for the luxury commodity increasing with $\varepsilon$, and then excess demand for the necessary commodity decreasing with $\varepsilon$. Therefore aggregate excess demand for the luxury commodity increases with both luxury parameters $\mathcal{\varepsilon}, \mathcal{E}^{\prime}$.

Proof. Consider the function $f(y ; a, \varepsilon, p, \bar{x}, \bar{y})=(\bar{x}-p(y-\bar{y}))^{a} y^{1-a} e^{(1-a) \varepsilon y}$ (problem 7), which represents the functions (1) constrained on budget lines, and whose maximization yields the Walrasian demand for the luxury commodity of the agent $(a, \mathcal{E})$ for initial endowment $(\bar{x}, \bar{y}) \in(0,1) \times(0,1)$. Being a function of the single variable $y \in(0,1), f$ is quasisupermodular (MS, p. 162). Being $\frac{\partial^{2} f}{\partial y \partial \varepsilon}>0$ in $(0,1) \times[0, \infty)$ for any $a \in(0,1), p \in(0, \infty)$ and $(\bar{x}, \bar{y}) \in(0,1) \times(0,1), f$ has increasing differences in $(y, \varepsilon)$ (MS, Theorem 6), and therefore satisfies the single crossing property (MS, p. 164). Then, by the fundamental monotonicity theorem (MS, Theorem 4), we are guaranteed that $\operatorname{argmax}_{y} f$ increases with $\varepsilon$ for any $a, p,(\bar{x}, \bar{y})$ in the proper intervals.

12 Compare the terminology employed by Afriat (1987) in the analysis of CD preferences, according to which the parameter $a$ measures “distribution of weights” of wants (ivi, p. 327), 


\section{ANDREA MANTOVI On Luxury and Equilibrium}

Figure A1 plots the function $f(y ; a, \varepsilon, p, \bar{x}, \bar{y})$ for a $=0.5,(\bar{x}, \bar{y})=(0.5,0.5)$ and sample values of $p$, and represents transparently the monotonicity of the luxury effect, which extends for all values of $\varepsilon$ : the luxury effect embodied by the utility functions (1) is a robust one.

Definitely, such a monotonicity does not hold for the individual distributive effect. Figure A2 plots the function $f$ for $\varepsilon=0.5,(\bar{x}, \bar{y})=(0.5,0.5)$ and sample values of $p$, and displays the transition from a phase in which $f$ increases with $a$ to a phase in which $f$ decreases with $a$, thereby ruling out the possibility of monotone individual distributive effects. Still, such effect does play a significant role in our economies, which can be transparently represented on normative grounds.

\section{Pareto sets}

The Pareto set of an exchange economy is the set of Pareto efficient allocations. The crucial result has long been established that any WE in an Edgeworth box with nonsatiated preferences belongs to the Pareto set (first welfare theorem), and interpreted as a consistent representation of the working of Adam Smith's “invisible hand”. Noticeably, capitalizing on the analytical tractability of our preferences, we are in a position to set forth explicit solutions for Pareto sets; as is well known, the equation for the Pareto set represents the condition of proportionality of the differentials (gradients) of the utility functions of the two agents. ${ }^{13}$

PROPOSITION 3. The equation of the Pareto set for our exchange economies reads

$$
\tilde{x}(y)=\frac{1}{1+\frac{(1-a) a^{\prime}}{a\left(1-a^{\prime}\right)} \frac{(1-y)(1+\varepsilon y)}{y\left(1+\varepsilon^{\prime}(1-y)\right)}}
$$

Proof. Writing respectively $U^{A}$ and $U^{B}$ for the utility functions of the agents A, B, the condition $d U^{A} \propto d U^{B}$ reads

$$
\frac{\frac{\partial U^{A}}{\partial y}}{\frac{\partial U^{A}}{\partial x}}=\frac{1-a}{a} \frac{x}{y}(1+\varepsilon y)=\frac{\frac{\partial U^{B}}{\partial y}}{\frac{\partial U^{B}}{\partial x}}=\frac{1-a^{\prime}}{a^{\prime}} \frac{(1-x)}{(1-y)}\left(1+\varepsilon^{\prime}(1-y)\right)
$$

i.e.

\footnotetext{
${ }^{13}$ Edgeworth (1881) himself pointed out the relevance of such an equation, as well as its fatherhood,
} "Professor Jevons's equation” (ivi, p. 21). 
Review of Economic Analysis 6 (2014) 87-118

$$
\frac{1-a}{a} x(1+\varepsilon y)(1-y)=\frac{1-a^{\prime}}{a^{\prime}}(1-x) y\left(1+\varepsilon^{\prime}(1-y)\right)
$$

from which we obtain (23).

Formula (23) generalizes the one set forth by Afriat (1987, p. 327) for CD economies in terms of the "spread" $M \equiv \frac{a\left(1-a^{\prime}\right)}{a^{\prime}(1-a)}$ between the "wants" of the CD agents. The simplicity of formula (23) witnesses the effectiveness of our model. The same limits

$$
\lim _{y \rightarrow 0} \tilde{x}(y)=0, \lim _{y \rightarrow 1} \tilde{x}(y)=1
$$

hold as for CD (and not for general) agents. It follows straightforwardly from formula (23) that the allocation $(\bar{x}=1 / 2, \bar{y}=1 / 2)$ is a WE for any pair of agents (1) such that

$$
\frac{a^{\prime}(1-a)}{a\left(1-a^{\prime}\right)} \frac{(1+0.5 \varepsilon)}{\left(1+0.5 \varepsilon^{\prime}\right)}=1
$$

As expected, such a condition is satisfied by identical agents. In fact, there exist many more pairs of agents for which (27) is satisfied, and then for which an equal initial allocation of resources is stable under exchange possibilities: for any given pair a, $a^{\prime} \in(0,1)$ there exist infinite nonnegative pairs $\varepsilon, \varepsilon^{\prime}$ such that (27) is satisfied. Still, such an occurrence has Lebesgue measure zero in the space $(0,1) \times[0, \infty)$ of our agents.

For the sake of graphical analysis, notice that for the agents Adam and Bob formula (23) reads

$$
\tilde{x}(y)=\frac{y(1+0.5(1-y))}{\frac{1}{3}(1+0.4 y)(1-y)+(1+0.5(1-y)) y}=\frac{y^{2}-3 y}{\frac{19}{15} y^{2}-\frac{13}{5} y-\frac{2}{3}}
$$

whose plot is given in Figure 6, against sample indifference curves. Evidently, the linear approximation of the Pareto set (23) about the corners is essential for qualitative analysis.

Definitely, the Taylor expansion of (23) about $y=0$ and $y=1$ is feasible, since for $a, a^{\prime}$ $\in(0,1)$ and nonnegative $\varepsilon$ and $\varepsilon^{\prime}$, the function (23) is $C^{\infty}$ in a neighbourhood of $[0,1]$. Notice in first instance that the denominator in (23) is positive at both $y=0$ and $y=1$. Then, suppose the denominator of (23) vanishes at $y \in(0,1)$. Then, by (24), one has $\frac{1-a^{\prime}}{a^{\prime}} y\left(1+\varepsilon^{\prime}(1-y)\right)=0$, which cannot occur for $y \in(0,1)$. Then, for any $a, a^{\prime}, \varepsilon$ and $\varepsilon^{\prime}$ in the 
proper intervals, there exists an open neighbourhood of $[0,1]$ in which $(23)$ is the ratio of $C^{\infty}$ functions with nonvanishing denominator, and is therefore $C^{\infty}$. Thus, we are guaranteed the possibility to Taylor expand the function (23) about the corners, and thereby obtain

LEMMA 1. The Taylor expansions of (23) about $y=0$ and $y=1$ result in

$$
\begin{aligned}
& \tilde{x}(h)=\frac{a\left(1-a^{\prime}\right)}{(1-a) a^{\prime}}\left(1+\varepsilon^{\prime}\right) h+O\left(h^{2}\right) \\
& \tilde{x}(1-h)=1-\frac{(1-a) a^{\prime}}{a\left(1-a^{\prime}\right)}(1+\varepsilon) h+O\left(h^{2}\right)
\end{aligned}
$$

Proof. Straightforward.

Figure 6

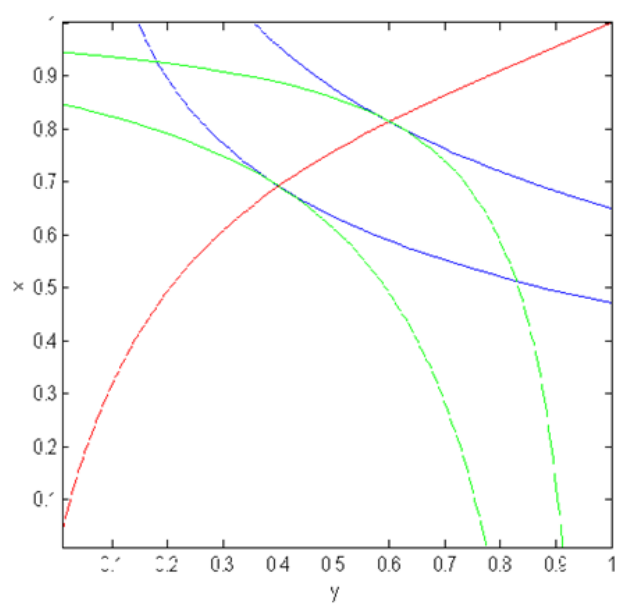

Pareto set for the agents Adam and Bob, against sample indifference curves.

Notice the symmetry linking the above pair of formulas: one can obtain one from the other by substituting the parameters of agent A with the parameters of agent B and vice-versa. Thus, we recover the well-known fact that for $\mathrm{CD}$ agents the angular coefficients at the corners are reciprocal, as well as for any pair of points symmetric with respect to the diagonal $x=1-y$. Furthermore, as expected, $\varepsilon\left(\varepsilon^{\prime}\right)$ has no first order effect in the neighbourhood of the lower 
(upper) corner, in that the preferences (1) approach the CD limit in the limit of vanishing $y$ : in the limit in which the endowment of agent $\mathrm{A}$ is vanishing, his preferences approach the CD limit, and the slope of the Pareto set is determined by $\frac{a\left(1-a^{\prime}\right)}{(1-a) a^{\prime}}$ and by the value of $\varepsilon^{\prime}$.

A natural question concerning the effects generated by $\varepsilon$ and $\varepsilon^{\prime}$ is as follows. Can we find WE allocations for $C D$ agents which are invariant with respect to the action of interplay luxury effects? That is, does the Pareto set $\tilde{x}_{a, a^{\prime}}(y)=\frac{y}{y+\frac{(1-a) a^{\prime}}{a\left(1-a^{\prime}\right)}(1-y)}$ for the CD agents $a$, $a^{\prime}$ intersect the Pareto set (23) for the agents $a, a^{\prime}, \mathcal{\varepsilon}, \mathcal{\varepsilon}^{\prime}$ ? The answer is a positive one.

LEMMA 2. For any pair of agents (1), the Pareto set (23) crosses the Pareto set $\tilde{x}_{a, a^{\prime}}(y)$ for the CD agents $a, a^{\prime}$.

Proof. On account of Lemma 1, the derivative of the function (23) is larger than the derivative of $\tilde{x}_{a, a^{\prime}}(y)$ in a neighbourhood of the lower corner, so that for small enough $y$ the Pareto curve (23) lies above the curve $\tilde{x}_{a, a^{\prime}}(y)$. On the other hand, the derivative of (23) is larger than the derivative of $\tilde{x}_{a, a^{\prime}}(y)$ in a neighbourhood of the upper corner as well, so that for $y$ close enough to 1 the curve (25) lies below $\tilde{x}_{a, a^{\prime}}(y)$. Then, the two curves must intersect an odd number of times; in fact, it is not difficult to convince oneself that such intersection occurs at a single point, since the rational functions (23) display at most a pair of changes of concavity.

Figure A5 displays an example of the phenomenon identified in Lemma 2. In fact, once the linear approximations to the Pareto set (23) are given at the corners, a qualitative description of its shape can be easily conjectured. In the limit of no effects, i.e. for $a-a^{\prime}=\varepsilon=\varepsilon^{\prime}=0$, one reduces to the straight Pareto set $\tilde{x}(y)=y$ for identical CD agents. With respect to such benchmark cases, let us define region $H$ of our boxes as the locus of points at which $x>y$, and region $L$ for the opposite inequality. It has been long established (see for instance Mas-Colell et al., 1995, p. 533) that for homothetic consumers the Pareto set cannot intersect both regions $H$ and $L$. Definitely, our model entails more structure, as established by 
ANDREA MANTOVI On Luxury and Equilibrium

PROPOSITION 4. Pareto sets for our economies are characterized by distributive and luxury effects as follows.

I) In the absence of effects, i.e. for $a-a^{\prime}=\varepsilon=\varepsilon^{\prime}=0$, the Pareto set coincides with the diagonal $x=y$.

II) In the absence of luxury effects, i.e. for $\varepsilon=\varepsilon^{\prime}=0$, the Pareto set has definite convexity and lies entirely in region $H$ for $a>a^{\prime}$ and entirely in region $L$ for $a<$ $a^{\prime}$

III) In the absence of interplay distributive effects and with individual luxury effects, i.e.

$a-a^{\prime}=\varepsilon=0, \varepsilon^{\prime}>0$, or $a-a^{\prime}=\varepsilon^{\prime}=0, \varepsilon>0$, the Pareto set has no definite convexity and lies entirely in region $H$ for $\varepsilon^{\prime}>0$, and entirely in region $L$ for $\varepsilon>$ 0 .

IV) In the absence of interplay distributive effects and with interplay luxury effects, i.e.

$a-a^{\prime}=0$ and $\varepsilon, \varepsilon^{\prime}>0$, the Pareto set has no definite convexity and intersects both regions $H$ and $L$.

$V)$ In the general case the Pareto set has no definite convexity and may or may not lie entirely in one region.

VI) If agents $A$ and $B$ are endowed with the same preferences, the Pareto curve is skew-symmetric with respect to the secondary diagonal $x=1-y$.

Proof. All points can be checked directly via formula (23). In fact, by I) and II) we recover well known properties of CD exchange economies (largely employed in the theory of trade) represented in Figure A3. Point III) isolates individual luxury effects: with the help of (29) one envisions the effect of $\varepsilon^{\prime}$ of increasing the slope of the Pareto curve about the lower corner above $45^{\circ}$ so that the curve lies entirely in region $H$ (Figure A4, upper). Correspondingly, $\varepsilon$ increases the slope of the Pareto curve about the upper corner above $45^{\circ}$, so that the curve lies entirely in region $L$ (Figure A4, lower). Point IV) is a consequence of Lemma 2, so that the Pareto set crosses the principal diagonal (Figure A5). Then, point V) enlarges the landscape to the general case, in which anything goes. To conclude, point VI) is an obvious symmetry requirement (Figure A6). 


\section{Conclusions}

It was the aim of the present contribution to employ a class of transcendental preferences for luxury in order to work out new solutions for competitive exchange equilibrium within the Edgeworth box. We have succeeded in computing Walrasian demand functions in both parametric and Cartesian forms, equilibrium prices and allocations, and then Pareto sets, in terms of simple combinations of elementary functions. Such transparent solutions enlighten the positive relevance of the luxury-necessity dichotomy in fixing up main features of well behaviour of exchange economies, in first instance, regularity, uniqueness of equilibrium and price tâtonnement stability. In fact, with the exception of the properties related to homotheticity, our economies retain the nice properties of Cobb-Douglas exchange economies. "For a theorist, the best of all possible worlds is one in which the social situation being analyzed can be formalized in a manner that, on the one hand, is very parsimonious [...] and, on the other hand, manages to predict a unique outcome” (Mas-Colell et al., 1995, p. 590). Our economies seem to fit such requirements. The relevance of our model for empirical analysis may rest on the smooth Cobb-Douglas limit of our economies.

Taking for granted the relevance of explicit solutions for general equilibrium models, we have been trying and enlighten the effectiveness of our solutions for exchange in providing transparent insights, which may complement standard technical perspectives in PTGE, with a sharp rationale for imposing structure on excess demand functions in terms of a luxury effect, along the lines discussed by Freixas and Mas-Colell (1987). In fact, the factor $F(y)=\exp (\varepsilon(1-a) y)$ generating the luxury effect in (1) can be employed in order to generate analogous effects with respect to any homothetic function, for instance $\mathrm{CES}^{14}$ models. Evidently, a natural generalization of the previous analysis can be obtained by enlarging the number of commodities subject to exchange; for instance one may inquire whether the conditions discussed by Freixas and Mas-Colell (1987) can be satisfied by suitable generalizations of the utility form (1). Noticeably, our model enables one to pair different agents, and consider any initial allocation (distribution of income).

The transcendental utility functions we have employing belong to the class of KingPlosser-Rebelo functions, which seem to represent a natural setting for the study of within-period labor supply as "an application of basic consumer theory" (Blundell and MaCurdy, 1999) to the representation of the leisure-consumption tradeoff; see Kimball and Shapiro (2008) and references therein.

The pervasive role of the Edgeworth box as building block of equilibrium arguments makes it difficult to envision a preferred line of progress for our results. For sure, the analytical tractability of our model may be applied to the analysis of

\footnotetext{
${ }^{14}$ Noticeably, CES preferences may lead to nonuniqueness of exchange equilibrium (Kehoe, 1998)
} 


\section{ANDREA MANTOVI On Luxury and Equilibrium}

distortionary taxes (see for instance Kehoe, 1998) and market failures in general. Application of our Edgeworth boxes to mechanism design (for instance along the lines tailored by Hurwicz and Reiter, 2006) may prove relevant. In addition, our model may be employed as a workhorse in the inquiry about the 'dark side' of exchange tailored by Hirshleifer (1994), for which Anderton and Carter (2008) provide a recent contribution which confines to homothetic agents; by their tractability, our solutions may represent a workhorse in the analysis of the allocation of resources to the defence of goods offered for trade.

Being the Edgeworth box an essential building block of the analysis of trade, applications of our explicit solution (23) for Pareto sets may pertain to factor allocations as shaped by the Stolper-Samuelson (1941) theorem, whose analysis is confined to homogeneous production functions. In addition, applications to the Rybczynski theorem and the Heckscher-Ohlin model of trade may represent interesting lines of inquiry.

Noticeably, as for the objects of trade, according to Afriat (1987), “These need not always be commodities, or bear a market price, or even be of a material kind at all. There is trade in liberty, justice, simplicity, and in favours; it takes place also with nature, or technology, or whatever there are allowances under a constraint” (ivi, p. 93). Needless to say, liberty and justice stand out in such respects. To conclude, thus, it is tempting to conjecture possible applications of our model to the philosophy of law tailored by Ronald Dworkin ${ }^{15}$ (2000), and in particular to the consequences of the principle of equality of resources as a foundational perspective on liberal philosophy. The luxury-necessity dichotomy defines quite general a logic of choice, to the extent that we consider choices as bivariate and such that, as wealth increases, the rational allocation of resources shifts monotonically towards 'luxury' in its broader sense, beyond the, albeit relevant, purchase of goods (think for instance of rational individuals arguing about allocating their spare time). In Dworkin's approach, economic mechanisms play a structural role, in first instance auctions and markets. "I argue that an equal division of resources presupposes an economic market of some form, mainly as an analytical device but also, to a certain extent, as an actual political institution” (ivi, p. 66). Possibly our model may represent a workhorse for the design of economic arguments, for which Edgeworth (1881) maintained that "He that will not verify his conclusions as far as possible by mathematics [...] will hardly realize the full value of what he holds" (ivi, p. 3). In such respects, the analytical tractability of our equilibrium model may well be taken as probe of its relevance.

\footnotetext{
${ }^{15}$ The most original and powerful philosopher of law in the English speaking world, according to the obituary published by the Guardian, Thursday 14 February 2013.
} 


\section{Appendix.}

The present appendix collects a number of figures meant to sharpen the insights developed in the main text. Figure A1 represents the monotonicity of the luxury effect: other things ( $a, p$ and initial endowment) equal, $\varepsilon$ drives an increase in utility for any $y$ along budget lines, and therefore an increase in Marshallian demand for the luxury commodity. Figure A2 shows that a corresponding property does not hold for the effect driven by $a$. Figure A3 to A6 picture sample Pareto sets meant to represent the phenomenology established in Proposition 4.

\section{FigureA1}
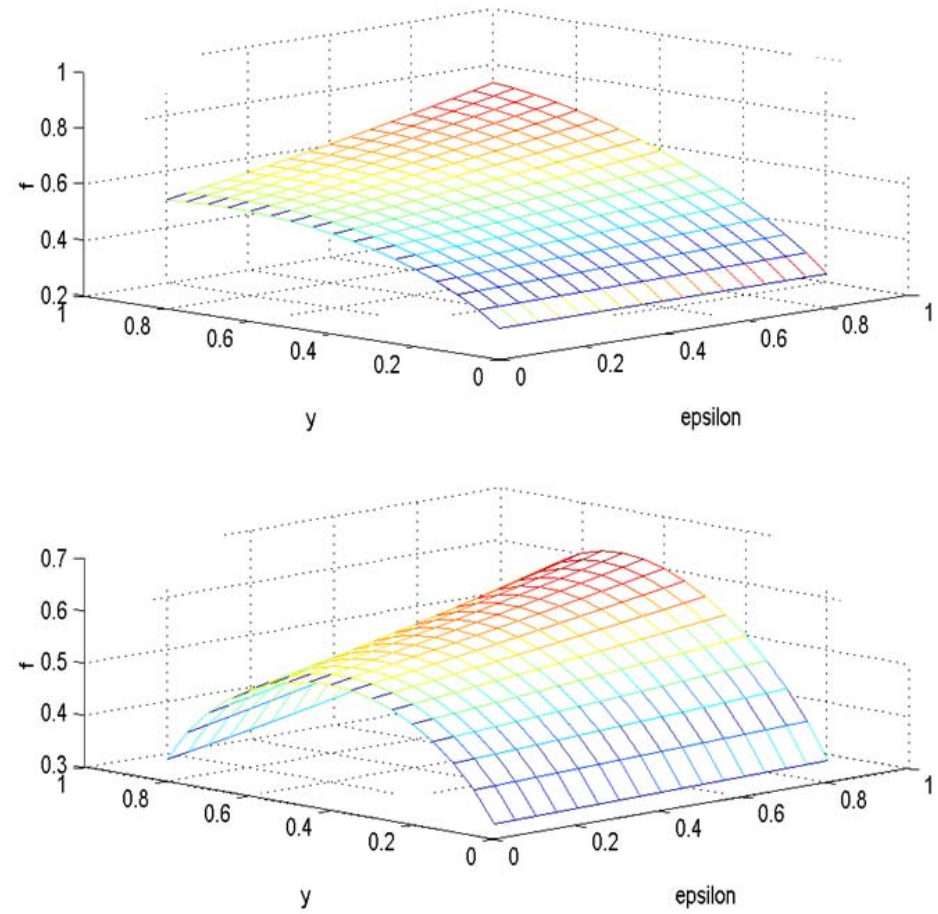

Above. Plot of the function $f(y ; a, \varepsilon, p, \bar{x}, \bar{y})=(\bar{x}-p(y-\bar{y}))^{a} y^{1-a} e^{(1-a) \varepsilon y}$ for $a=0.5,(\bar{x}=1 / 2, \bar{y}=1 / 2)$, and $p=0.5$. The monotonicity in $y$ signals that the budget line is nowhere tangent to indifference curves.

Below. Plot of the same function for $p=1$. For given $\varepsilon$, $\operatorname{argmax}_{y} f$ corresponds to the unique point at which the budget line is tangent to an indifference curve. 


\section{ANDREA MANTOVI On Luxury and Equilibrium}

Figure A2
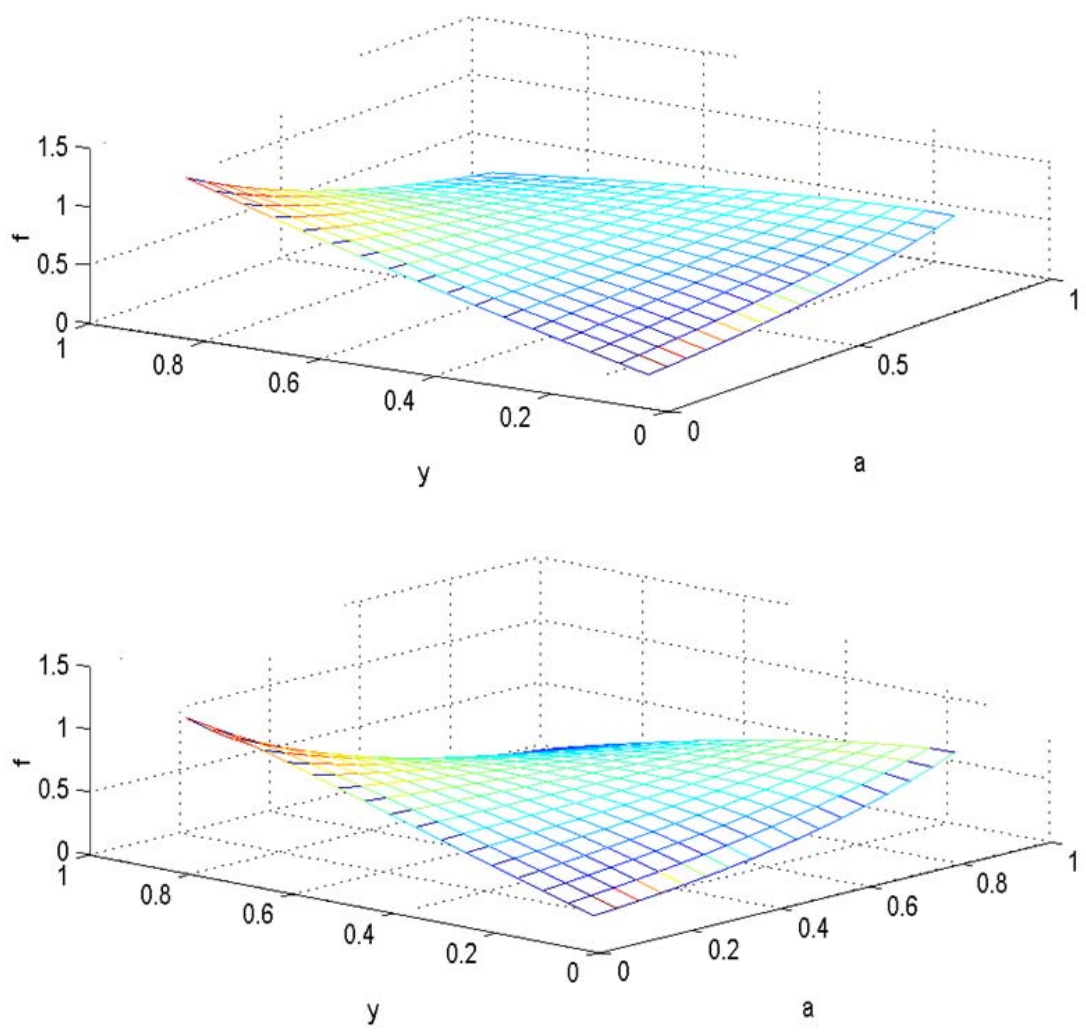

Plot of the function $f(y ; a, \varepsilon, p, \bar{x}, \bar{y})=(\bar{x}-p(y-\bar{y}))^{a} y^{1-a} e^{(1-a) \varepsilon y}$ for $\varepsilon=0.5$, $(\bar{x}=1 / 2, \bar{y}=1 / 2)$, and $p=0.5$ (above) and $p=1$ (below). 
Review of Economic Analysis 6 (2014) 87-118

Figure A3
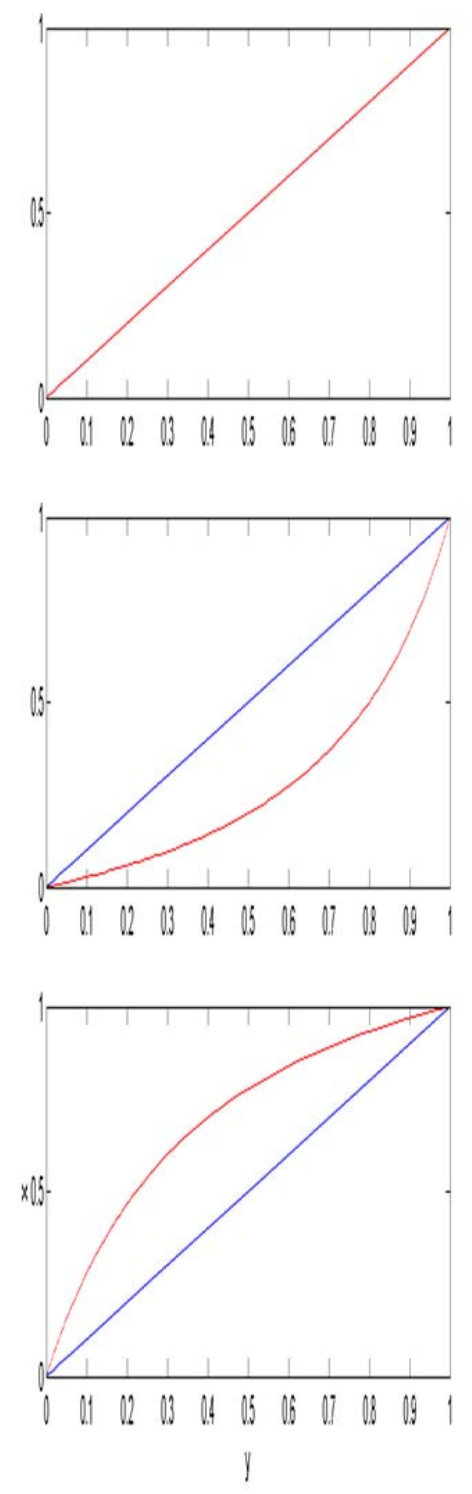

Sample Pareto sets for our economies in the absence of luxury effects, for the $\mathrm{CD}$ agents $\left(a, a^{\prime}\right)=(0.2,0.2$; upper $),(0.2,0.5$; middle $),(0.6,0.3,0,0$; lower). 


\section{ANDREA MANTOVI On Luxury and Equilibrium}

Figure A4
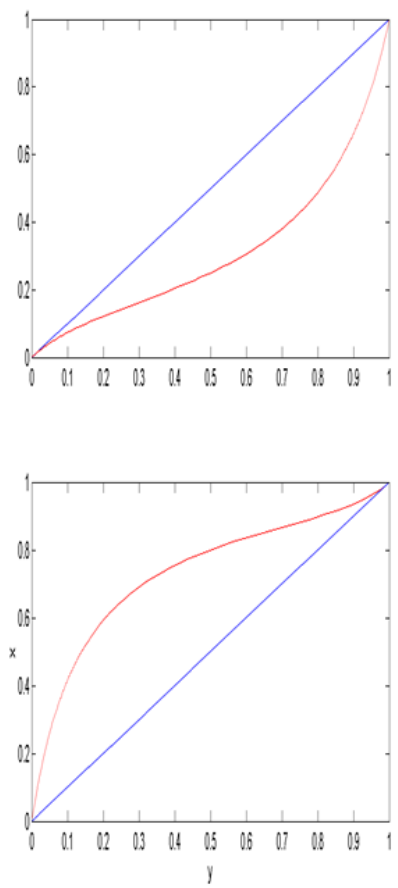

Pareto sets for $\left(a, a^{\prime}, \varepsilon, \varepsilon^{\prime}\right)=(0.2,0.2,4,0$; upper $),(0.2,0.2,0,6$; lower $)$.

Figure A5

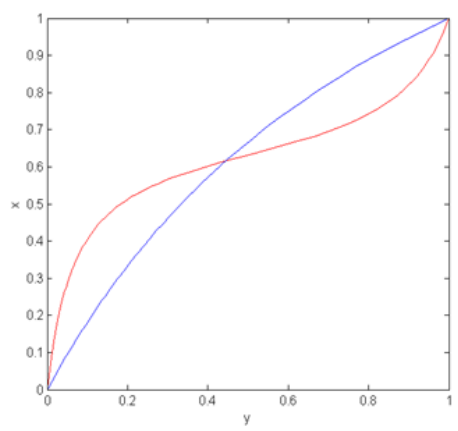

The Pareto set $\tilde{x}_{0.2,0.33}(y)$ (concave curve) against the Pareto set for ( $a, a^{\prime}, \mathcal{\varepsilon}$, $\left.\varepsilon^{\prime}\right)=(0.2,0.33,5,4)$. 
Review of Economic Analysis 6 (2014) 87-118

Figure A.6

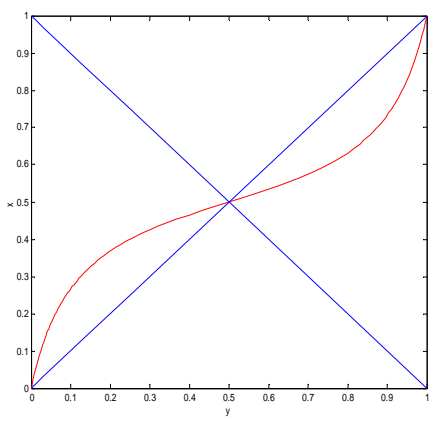

The skew symmetric Pareto set for the identical agents $\left(a, a^{\prime}, \varepsilon, \varepsilon^{\prime}\right)=(0.2$, $0.2,4,4)$.

\section{References}

Acemoglu, D. (2008), Modern Economic Growth, Princeton: Princeton University Press Afriat, S. (1987), Logic of Choice and Economic Theory, Oxford: Clarendon Press

Aitchison, J., and J. Brown (1954), A Synthesis of Engel Curve Theory.” Review of Economic Studies, 22(1), 35-46.

Anderton, C., and J. Carter (2008), Vulnerable trade: The dark side of an Edgeworth box. Journal of Economic Behavior and Organization, 68(2), 422-432.

Arrow, K., and G. Debreu (1954), Existence of an Equilibrium for a Competitive Economy. Econometrica, 22(3), 265-290.

Arrow, K., Block, H., and L. Hurwicz (1959), On the Stability of the Competitive Equilibrium II. Econometrica, 27(1), 82-109.

Balasko, Y., and M. Tvede (2010), General equilibrium without utility functions: how far to go? Economic Theory, 45(1-2), 201-225.

Binmore, K. (2007), Playing for Real. Oxford: Oxford University Press.

Blundell, R., and T. MaCurdy (1999), Labor Supply: a Review of Alternative Approaches. In Handbook of Labor Economics, Vol. IIIA, Ashenfelder, O. and Card, D. eds., North Holland.

Chakrabarty, M., and W. Hildenbrand (2011), Engel's Law Reconsidered. Journal of Mathematical Economics, 47(3), 289-299.

Chambers, R. (1988), Applied production analysis. Cambridge: Cambridge University Press.

Chambers, R., and T. Mitchell (2001), Homotheticity and Non-Radial Changes. Journal of Productivity Analysis, 15(1), 31-39. 


\section{ANDREA MANTOVI On Luxury and Equilibrium}

Chiappori, P. (1985), Distribution of Income and the "Law of Demand", Econometrica, 53(1), 109-127.

Cowell, F. (2005), Microeconomics. Principles and Analysis. Oxford: Oxford University Press.

Deaton, A. and J. Muellbauer (1980), An Almost Ideal Demand System. American Economic Review, 70, 312-326.

Debreu, G. (1976), Regular Differentiable Economies. American Economic Review, 66(2), 280-287.

de Janvry, A. (1972), The Class of Generalized Power Production Functions, American Journal of Agricultural Economics, 54(2), 234-237.

Dworkin, R. (2000), Sovereign Virtue. Harvard: Harvard University Press.

Edgeworth, F. (1881), Mathematical Psychics. London: C. Kegan Paul \& Co.

Freixas, X., and A. Mas-Colell. Engel Curves Leading to the Weak Axiom in the Aggregate. Econometrica, 55(3), 1987, 515-531.

Fuss, M., D. McFadden, and Y. Mundlak (1978), A Survey of Functional Forms in the Economic Analysis of Production. In Production Economics, Vol. I, Fuss, M. and McFadden, D. eds.

Jaffé, W. (1967), Walras' Theory of Tâtonnement. A Critique of Recent Interpretations. Journal of Political Economy, 75(1), 1-19.

Halter, A., Carter, H., and J. Hocking (1957), A Note on the Transcendental Production Function $\mathrm{y}=\mathrm{cx} 1^{\mathrm{a} 1} \mathrm{e}^{\mathrm{b} 1 \times 1} \times 2^{\mathrm{a} 2} \mathrm{e}^{\mathrm{b} 2 \times 2}$, American Journal of Agricultural Economics, 39(4), 966974.

Hildenbrand, W. (1983), On the "Law of Demand”. Econometrica, 51(4), 997-1019.

Hirshleifer, J. (1994), The Dark Side of the Force. Economic Inquiry, 32(1), 1-10.

Hurwicz, L., and S. Reiter (2006), Designing Economic Mechanisms. Cambridge: Cambridge University Press.

Kehoe, T. (1998), Uniqueness and Stability. In Elements of General Equilibrium Analysis. Basil: Blackwell.

Kihlstrom, R., Mas-Colell, A., and H. Sonnenschein (1976), The Demand Theory of the Weak Axiom of Revealed Preference. Econometrica, 44(5), 971-978.

Kimball, M. and M. Shapiro (2008), Labor Supply: Are the Income and Substitution Effects Both Large or Both Small? NBER Working Paper No. 14208.

Mantovi, A. (2013a), On the Commutativity of Expansion and Substitution Effects. Journal of Economics, 110(1), 83-105.

Mantovi, A. (2013b), Differential duality. Working paper EP05, Dipartimento di Economia, Università degli Studi di Parma.

Mas-Colell, A. (1991), On the uniqueness of equilibrium once again. In W. A. Barnett et al., eds, Equilibrium Theory and Applications. Cambridge University Press. 
Review of Economic Analysis 6 (2014) 87-118

Mas-Colell, A., Whinston, M., and J. Green (1995), Microeconomic Theory. Oxford: Oxford University Press.

Milgrom, P., and C. Shannon (1994), Monotone Comparative Statics, Econometrica, 62(1), 157-180.

Mundlak, Y. (1964), Transcendental Multiproduct Production Functions. International Economic Review, 5(3), 273-284.

Quah, J. (2003), Market demand and comparative statics when goods are normal. Journal of Mathematical Economics, 39(3-4), 317-333.

Samuelson, P. (1938), A Note on the Pure Theory of Consumer's Behavior. Economica New Series, 5, 61-71.

Samuelson, P., and S. Swamy (1976), Invariant Economic Index Numbers and Canonical Duality: Survey and Synthesis. American Economic Review, 64(4), 566-593.

Sato, R. (1976), Self-Dual Preferences. Econometrica, 44(5), 1017-1032.

Sato, R. (1981), Theory of Technical Change and Economic Invariance. New York: Academic Press.

Silberberg, E. (2008), Hicksian and Marshallian demands. The New Palgrave Dictionary of Economics.

Silva, J., and M. Toledo (2013), The Unemployment Volatility Puzzle: the Role of Matching Costs Revisited. Economic Inquiry, 51(1), 836-843.

Sonnenschein, H. (1972), Market Excess Demand Functions. Econometrica, 40(3), 549-563.

Stolper, W., and P. Samuelson (1941), Protection and Real Wages. Review of Economic Studies, 9(1), 58-73.

Uzawa, H. (1960), Walras' Tâtonnement in the Theory of Exchange. Review of Economic Studies, 27(3), 182-194.

Varian, H. (1992), Microeconomic Analysis. Third Edition. Norton.

Walras, L. (1974), Elements d'economie politique pure. Lausanne, Switzerland. 